\title{
Soft mode behavior and evidence for pressure-induced magnetostructural effects in $\operatorname{Pr}_{2} \mathrm{O}_{3}$
}

\author{
J. E. Slimak $\odot,{ }^{1}$ A. Sethi, ${ }^{1}$ T. Kolodiazhnyi, ${ }^{2}$ and S. L. Cooper $\oplus^{1}$ \\ ${ }^{1}$ Department of Physics and Materials Research Laboratory, University of Illinois, Urbana, Illinois 61801, USA \\ ${ }^{2}$ National Institute for Materials Science, 1-1 Namiki, Tsukuba, Ibaraki 305-0044, Japan
}

(Received 13 July 2020; accepted 1 October 2020; published 30 October 2020)

\begin{abstract}
The energetic proximity of crystal electric field (CEF) to phonon excitations in rare-earth materials can lead to strong electron-phonon coupling, exotic behavior, and technologically useful properties. We present a temperature-, pressure-, and magnetic field-dependent Raman scattering study of $\operatorname{Pr}_{2} \mathrm{O}_{3}$ that reveals soft phonon mode behavior and anomalies associated with CEF excitations, which are indicative of strong phonon-CEF coupling. We also show that both an applied magnetic field and pressure induce a similar splitting of the doubly degenerate $\Gamma_{3}$ crystal-field level, providing evidence for pressure-induced magnetostructural effects in $\operatorname{Pr}_{2} \mathrm{O}_{3}$. These results demonstrate the importance of low-lying CEF excitations and strong CEF-phonon coupling in governing the phases and properties of rare-earth materials.
\end{abstract}

DOI: 10.1103/PhysRevResearch.2.043169

\section{INTRODUCTION}

The energetic proximity of phononic states to the atomiclike $f$-electronic crystal-electric-field (CEF) levels in rare earth materials is conducive to strong electron-phonon coupling that can give rise to interesting phenomena and novel material properties. Previous studies of rare earth materials have discovered examples of Davydov splitting [1], phase transitions induced by the cooperative Jahn-Teller effect [2-4], and coupled vibronic modes with mixed electronic and phononic character [1,5-8]. Mixing of phononic and electronic states in rare-earth materials can have a significant impact on the functional properties [8,9]. For example, recent Raman scattering measurements revealed that the onset of a giant magnetodielectric response and Néel ordering in $\mathrm{Ce}_{2} \mathrm{O}_{3}$ is associated with emergent vibronic modes, i.e., coupled phonon and crystal electric field excitations [8]. These results suggest a novel mechanism for magnetoresponsive behavior-namely, a field-tunable coupling between electronic and phononic degrees of freedom - that may be prevalent in rare-earth materials.

The rare-earth (RE) sesquioxide family, $\mathrm{R}_{2} \mathrm{O}_{3}(\mathrm{R}=$ rare-earth), offer an opportunity to explore the role CEFphonon coupling plays in governing the physical properties of rare-earth materials. The RE sesquioxide family has been investigated extensively as constituents in layered heterostructures for microelectronics and catalytic systems. However, the microscopic mechanisms that give rise to the interesting physical properties of these materials have been relatively unexplored.

Published by the American Physical Society under the terms of the Creative Commons Attribution 4.0 International license. Further distribution of this work must maintain attribution to the author(s) and the published article's title, journal citation, and DOI.
$\mathrm{Pr}_{2} \mathrm{O}_{3}$ is a particularly interesting system in which to study interactions between crystal electric field (CEF) excitations and phonons. Each $\mathrm{Pr}^{3+}$ ion hosts two valence $f$-electrons, which gives rise to a rich set of low-energy CEF excitations associated with the ${ }^{3} \mathrm{H}_{4}$ multiplet. While most of the RE sesquioxides crystallize in the cubic bixbyite structure, the $R=\mathrm{La}, \mathrm{Ce}, \mathrm{Pr}$, and $\mathrm{Nd}$ members of the series exist as trigonal, $P \overline{3} 2 / m 1$ crystals. The local CEF that the $\operatorname{Pr}^{3+}$ ions experience splits the ground state electronic multiplet into six distinct levels, three singly degenerate and three doubly degenerate excitations. The presence of additional crystal field levels in $\operatorname{Pr}_{2} \mathrm{O}_{3}$ affords a richer environment for CEF-phonon coupling compared to $\mathrm{Ce}_{2} \mathrm{O}_{3}$, making $\mathrm{Pr}_{2} \mathrm{O}_{3}$ an exciting setting to search not only for emergent vibronic excitations, but also for other realizations of electron-phonon coupling.

In this paper, we present the first observations of CEF excitations in $\operatorname{Pr}_{2} \mathrm{O}_{3}$ at low temperature and study the temperature, pressure, and magnetic field dependencies of the Ramanactive CEF and phonon excitations. Interestingly, we observe temperature-dependent softenings of the $A_{1 g}$ stretching and $E_{g}$ bending phonons that are correlated with anomalous temperature dependencies of the CEF excitations. We also show that both an applied magnetic field and pressure induce a similar splitting of the doubly degenerate $\Gamma_{3}$ CEF level, offering evidence for pressure-dependent magnetostructural effects in $\operatorname{Pr}_{2} \mathrm{O}_{3}$. The pressure-induced behavior is similar to that observed in Pr metal [10] and further demonstrates the importance of phonon-CEF coupling to the properties of $\operatorname{Pr}_{2} \mathrm{O}_{3}$.

\section{EXPERIMENTAL}

$\mathrm{Pr}_{2} \mathrm{O}_{3}$ was prepared from $99.9 \%$ pure $\mathrm{Pr}_{6} \mathrm{O}_{11}$ sourced from Wako, Japan. The raw powder was pressed into a 7-mm diameter disk and sintered at $1500^{\circ} \mathrm{C}$ for $6 \mathrm{~h}$ in pure hydrogen at a flow rate of $200 \mathrm{~cm}^{3} \mathrm{~min}^{-1}$. The furnace was 
connected to a glove box. All sample handling and postsinter preparation was done under an Ar atmosphere with an oxygen concentration of $0.4 \mathrm{ppm}$ and a water vapor dew point of $-77^{\circ} \mathrm{C}$. The sintered ceramics had a relative density of $95 \%$ and a light-green color. Phase purity was confirmed by powder X-ray diffraction (PXRD) (Rigaku Miniflex 600 $\mathrm{X}$-ray diffractometer, Japan). PXRD measurements indicated that $\operatorname{Pr}_{2} \mathrm{O}_{3}$ crystallizes in the $A$-type hexagonal structure having the $\mathrm{P} \overline{3} 2 / \mathrm{m} 1$ space group that is isostructural to $A$-type $\mathrm{Ce}_{2} \mathrm{O}_{3}$. The room-temperature lattice parameters of $a=b=$ 3.86032(5) $\AA$ and $c=6.01669(8) \AA$ were determined from a Le Bail refinement of the PXRD profile. For Raman studies, the sample was polished with a fine $\mathrm{SiC}$ abrasive paper under Ar.

Raman scattering measurements were performed using the 647.1-nm excitation line of a $\mathrm{Kr}^{+}$laser. To limit laser heating of the sample at low temperatures, the laser spot was focused to approximately $50 \mu \mathrm{m}$ in diameter and the incident laser power was maintained below $3 \mathrm{~mW}$. Scattered light was collected in a backscattering geometry, dispersed through a triple stage spectrometer, and then recorded with a liquid nitrogen-cooled charge-coupled device (CCD) detector. Samples were held in a continuous He-flow cryostat, and horizontally mounted in the open bore of a superconducting magnet. This experimental arrangement allows Raman scattering measurements to be conducted under simultaneous control of the temperature $(3-300 \mathrm{~K})$, magnetic field (0-9 T), and pressure (0-100 kbar) applied to the sample. All magnetic field-dependent measurements were performed in the Faraday geometry with the incident light wave vector parallel to the magnetic field, allowing the observation of splitting of magnetically active excitations despite the polycrystalline nature of our samples [11]. The incident light was circularly polarized and scattered light of all polarizations was collected. High pressure measurements were performed using a miniature cryogenic diamond anvil cell (MCDAC) to exert quasihydrostatic pressure on the sample via an argon pressure-transfer medium. The high-pressure cell was inserted into the cryostat, allowing the pressure to be changed in situ at low temperatures without any additional warming/cooling procedure. The pressure was determined from the shift in the fluorescence line of a ruby chip loaded in the cell along with the sample using the calibration of Dewaele et al. (2008) [12]. While the pressure applied to the sample by the MCDAC is quasihydrostatic, we expect that there are small pressure anisotropic pressure variations within the MCDAC.

The Raman spectra measured in this study were fitted with sets of Lorentzian lineshapes to obtain peak center energies and peak widths utilized for temperature, pressure, and magnetic field-dependent analyses. In cases where a peak split into two features as a function of pressure or magnetic field, the peaks were fit with two Lorentzians only where it was clearly evident that the splitting was present; for some temperature and pressure data points, this means that some features could be fit with either one or two Lorentzian peaks. In cases where a single Lorentzian peak provides a fit similar to or better than that provided by two Lorentzian peaks, the analysis presented in this paper utilizes the single Lorentzian fit to avoid overfitting of the data.

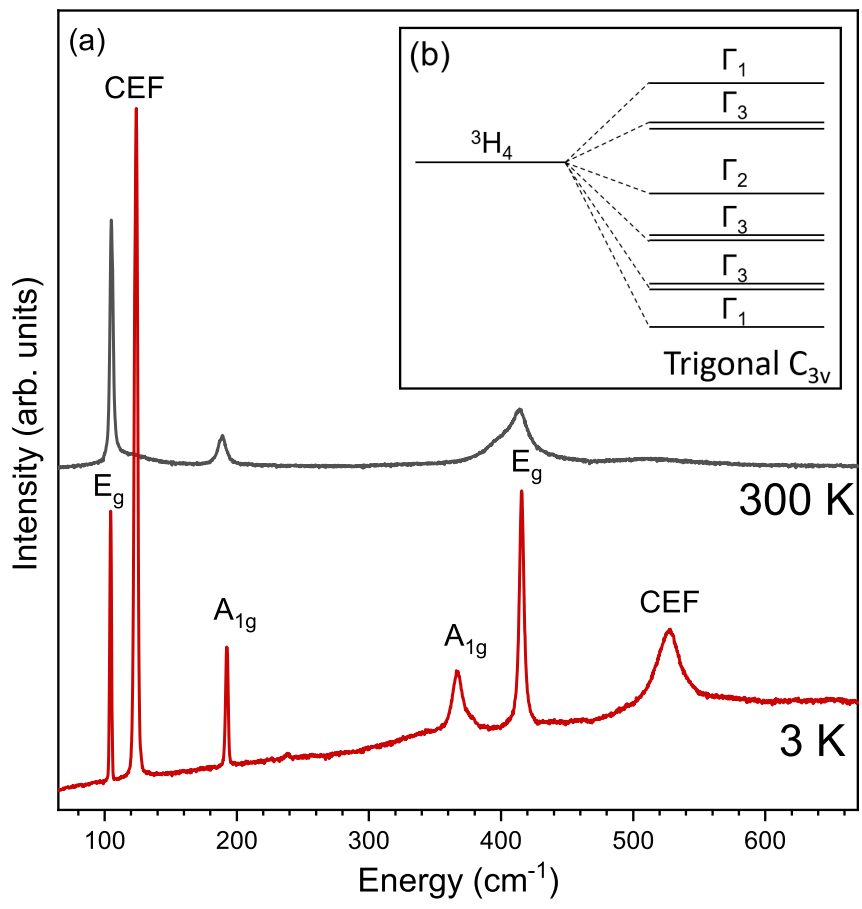

FIG. 1. (a) Composite spectra of the low energy excitations of $\mathrm{Pr}_{2} \mathrm{O}_{3}$, at 3 and $300 \mathrm{~K}$, between 65 and $670 \mathrm{~cm}^{-1}$. At low temperature, several new features appear compared to the room temperature spectrum, which we identify as CEF excitations. Additionally at low temperature, the narrowly separated stretching phonon modes become widely spaced in energy, primarily due to softening of the $A_{1 g}$ stretching phonon mode. Known phonon symmetries are included above the features in the $3-\mathrm{K}$ plot along with identification of the CEF excitations. (b) A proposed CEF splitting scheme based on measurements from [13-16].

\section{RESULTS AND DISCUSSION}

\section{A. Phonons and CEF excitations in $\mathrm{Pr}_{2} \mathrm{O}_{3}$}

Group theory predicts four Raman active phonons for $A$ type $\mathrm{Pr}_{2} \mathrm{O}_{3}$, with symmetries $2 A_{1 g}+2 E_{g}[17,18]$. We observe all four Raman-active phonons in $\mathrm{Pr}_{2} \mathrm{O}_{3}$ at room temperature [Fig. 1(a), black/upper spectrum]: the two lower energy modes with energies of 105.2 and $188.9 \mathrm{~cm}^{-1}$ are bending vibrations of the $\mathrm{Pr}-\mathrm{O}$ (II) bonds with $E_{g}$ and $A_{1 g}$ symmetries, respectively, while the two higher energy modes with energies of 396 and $414 \mathrm{~cm}^{-1}$ are stretching vibrations of the same bonds with $A_{1 g}$ and $E_{g}$ symmetries, respectively $[17,18]$.

In addition to the phonon modes, at low temperatures, we observe excitations at 124 and $527 \mathrm{~cm}^{-1}$ (Fig. 1, red/lower spectrum) which we identify as crystal electric field (CEF) excitations associated with transitions among the electronic levels of the $\operatorname{Pr}^{3+}{ }^{3} H_{4}$ manifold. The temperature-dependent intensities of these excitations, seen clearly in Fig. 2, are consistent with the increasing population of the electronic ground state with decreasing temperature. The crystal field Hamiltonian associated with the $C_{3 v}$ site symmetry of the $\operatorname{Pr}^{3+}$ ions in $\mathrm{Pr}_{2} \mathrm{O}_{3}$ is given by

$$
H_{\mathrm{CEF}}=B_{2}^{0} O_{2}^{0}+B_{4}^{0} O_{4}^{0}+B_{4}^{3} O_{4}^{3}+B_{6}^{0} O_{6}^{0}+B_{6}^{3} O_{6}^{3}+B_{6}^{6} O_{6}^{6}
$$




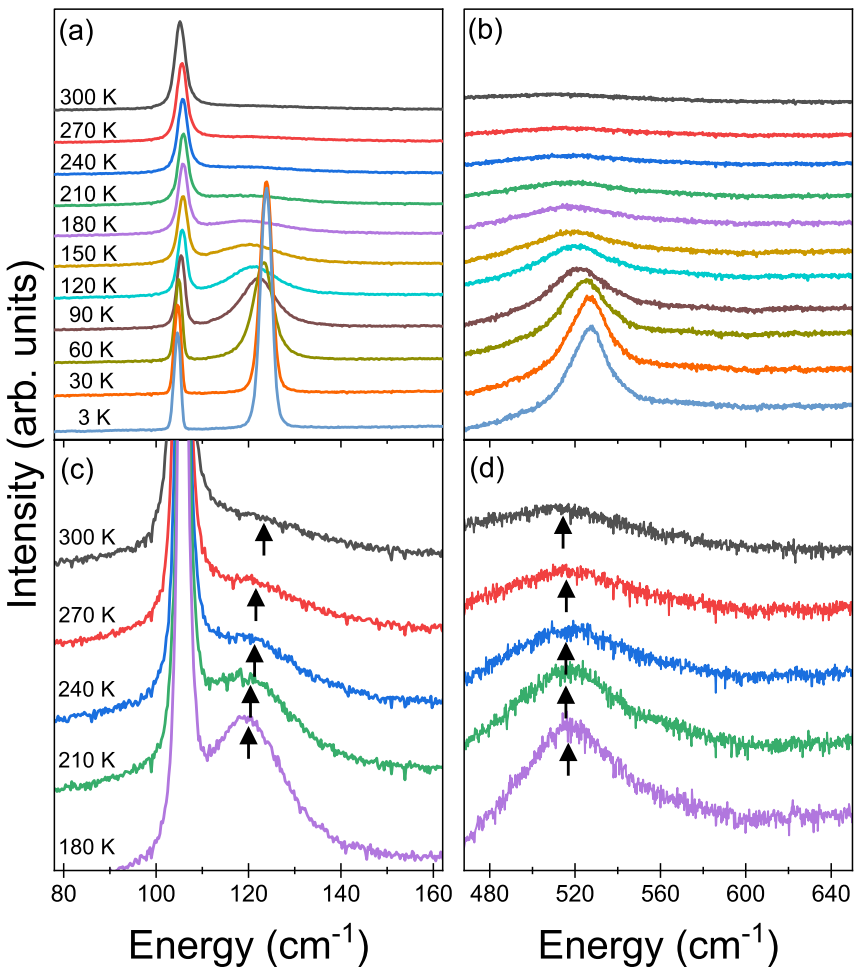

FIG. 2. Plot of the temperature dependent Raman intensities of the [(a) and (c)] lower energy and [(b) and (d)] higher energy CEF excitations. Arrows are included in the high temperature plots [(c) and (d)] to assist in the location of the peak center for the CEF excitations. In the plots of the full temperature range [(a) and (b)], the CEF excitations sharpen and increase in intensity greatly, becoming clearly visible below $180 \mathrm{~K}$. Individual spectra have been offset in the $y$-axis direction for clarity and each subfigure features spectra which all share the same $y$-axis scale, though the $y$-axis scale is not preserved between subplots: (c) and (d) feature a vertically enlarged view to more clearly indicate the position of the CEF excitations even when they have very low intensities.

where the $B_{m}^{n}$ are the crystal field parameters associated with the $O_{m}^{n}$ Stevens operators [13]. $H_{\mathrm{CEF}}$ splits the free ion ground state manifold of $\mathrm{Pr}^{3+}$ into three doublet and three singlet levels, $\Gamma_{\mathrm{CEF}}=2 \Gamma_{1}+\Gamma_{2}+3 \Gamma_{3}[13-16]$ [shown in Fig. 1(b)] and the eigenstates of $H_{\mathrm{CEF}}$ are given by linear combinations of the $J_{z}$ eigenstates, $A|n\rangle+B|n+3\rangle+C|n-3\rangle$ for $n \in\{-1,0,1\}$.

The excitation at $124 \mathrm{~cm}^{-1}$ is expected to be an excitation between the $\Gamma_{1} \mathrm{CEF}$ ground state to the first excited $\Gamma_{3}$ doublet of CEF states [13] given by linear combinations of $J_{z}$ eigenstates $A^{\prime}| \pm 4\rangle+B^{\prime}| \pm 1\rangle+C^{\prime}|\mp 2\rangle$ having $\Gamma_{3}$ symmetry. This first CEF doublet is expected to split in the presence of a magnetic field [13] because of the opposite angular momenta associated with the two degenerate first excited CEF levels. Indeed, Fig. 3(a), clearly shows a splitting of the doublet CEF excitation centered at $124 \mathrm{~cm}^{-1}$ into two distinct excitations, with an energy separation that increases linearly with magnetic field strength [Fig. 3(b)], confirming that the first excited CEF state is the doubly degenerate $\Gamma_{3}$ state. The magnitudes of the magnetic field dependencies of the energies of the two excitations are slightly different, with $\frac{\partial \omega}{\partial B}=-0.223 \pm 0.007$ and $0.264 \pm 0.014 \mathrm{~cm}^{-1} \mathrm{~T}^{-1}$ for
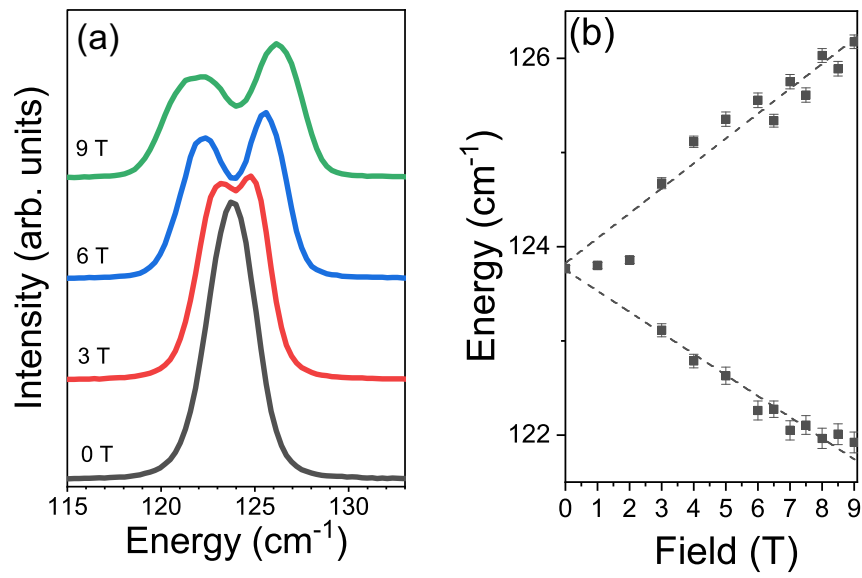

FIG. 3. (a) Plot of the magnetic field dependence of the Raman intensities of the lower energy CEF excitation, clearly showing its evolution from a doublet excitation into two distinct excitations at high field at $T=3 \mathrm{~K}$. (b) Summary plot of the field dependence of the peak-center energies of the lower energy CEF excitations at $T=3 \mathrm{~K}$. Linear fits of the energies of the two singlet excitations are included as guides to the eye.

the lower and higher energy excitations, respectively. We do not observe a field-induced splitting of the $\omega=527 \mathrm{~cm}^{-1}$ excitation. However, we cannot rule out the possibility that a splitting is not resolvable at the magnetic field strengths applied, so we are unable to confirm or rule out that the second excited state is also a doubly degenerate $\Gamma_{3}$ state as predicted [14-16].

The energies of the CEF transitions we observe at $T=3 \mathrm{~K}$ are consistent with calculations based on crystal field parameters obtained by fitting previous heat capacity measurements [13], magnetic susceptibility measurements [15,16], and optical absorption spectroscopy measurements [14].

\section{B. Temperature-dependent excitation spectra: evidence for phonon-CEF coupling in $\operatorname{Pr}_{2} \mathrm{O}_{3}$}

Figure 4 shows that the peak positions of the $105 \mathrm{~cm}^{-1} E_{g}$ bending and the $396 \mathrm{~cm}^{-1} A_{1 g}$ stretching phonon modes exhibit anomalous temperature dependencies. Specifically, we observe that the $E_{g}$ bending phonon mode initially increases in energy between 300 and $210 \mathrm{~K}$, then decreases in energy below $135 \mathrm{~K}$, while the $A_{1 g}$ stretching phonon mode softens by approximately $30 \mathrm{~cm}^{-1}$ as the temperature is reduced from 300 to $3 \mathrm{~K}$.

In addition to exhibiting temperature dependent mode softening, the 105 and $396 \mathrm{~cm}^{-1}$ phonons in $\operatorname{Pr}_{2} \mathrm{O}_{3}$ exhibit anomalous temperature dependent linewidths. Figure 5(a) shows that the linewidth of the $E_{g}$ bending phonon exhibits anomalous temperature-dependent broadening with increasing temperature. Additionally, Fig. 5(b) shows the nonmonotonic temperature-dependence of the $A_{1 g}$ stretching phonon linewidth, which is anomalous compared to that of the $E_{g}$ stretching phonon and cannot be described by a twophonon decay model [19]. The deviation from the typical two-phonon decay model for the $E_{g}$ bending phonon and the $A_{1 g}$ stretching phonon implies the existence of additional decay channels, and therefore additional interactions be- 

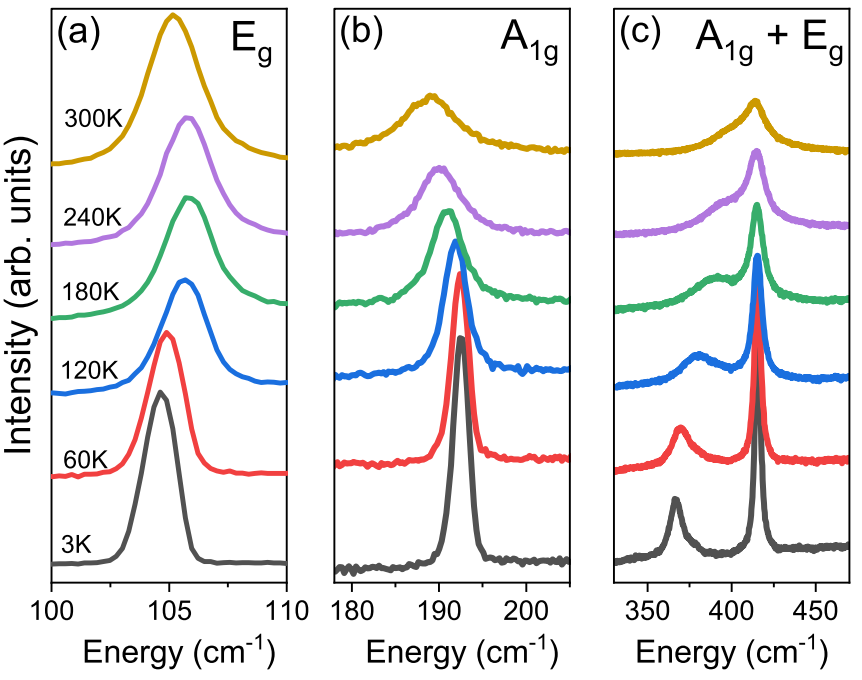

FIG. 4. Plot of temperature dependencies of Raman intensities for zone-center phonons from 3 to $300 \mathrm{~K}$. (a) The $E_{g}$ bending phonon displays nonmonotonic temperature dependence for its peakcenter energy. (b) The $A_{1 g}$ bending phonon hardens with decreasing temperature. (c) The $A_{1 g}$ stretching mode (lower energy) softens significantly with decreasing temperature while the $E_{g}$ stretching mode (higher energy) remains nearly constant in energy. Individual spectra have been offset in the $y$-axis direction for clarity and each subfigure features spectra which all share the same $y$-axis scale, though the $y$-axis scale is not preserved between subfigures.

tween phonons and other degrees of freedom, beyond typical anharmonicity.

Similar to the phonons in $\operatorname{Pr}_{2} \mathrm{O}_{3}$, the energies of the CEF excitation in $\mathrm{Pr}_{2} \mathrm{O}_{3}$ also exhibit anomalous temperature dependencies. The energy of the $124-\mathrm{cm}^{-1} \mathrm{CEF}$ excitation has a
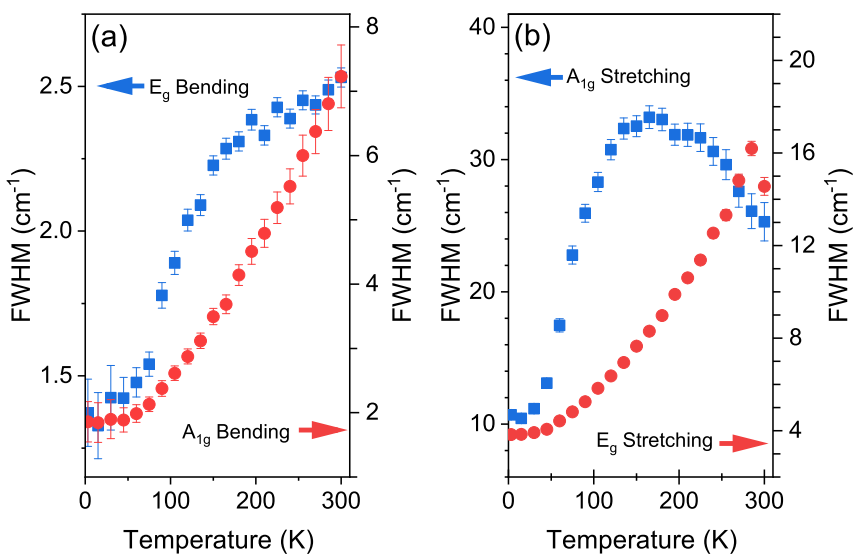

FIG. 5. Plots of the full-width at half maximum (FWHM) of the Raman active phonon modes in $\operatorname{Pr}_{2} \mathrm{O}_{3}$. (a) The FWHM of the $E_{g}$ (left axis, blue squares) bending phonon displays an unusual temperature dependence, while the $A_{1 g}$ bending phonon (right axis, red circles) narrows at lower temperature as would be expected for a phonon that decays via two-phonon relaxation channels. (b) The $A_{1 g}$ stretching phonon (left axis, blue squares) anomalously broadens as the temperature is decreased, before eventually narrowing below $165 \mathrm{~K}$, though the energetically proximate $E_{g}$ stretching phonon (right axis, red circles) narrows in accordance to the model of two-phonon decay.
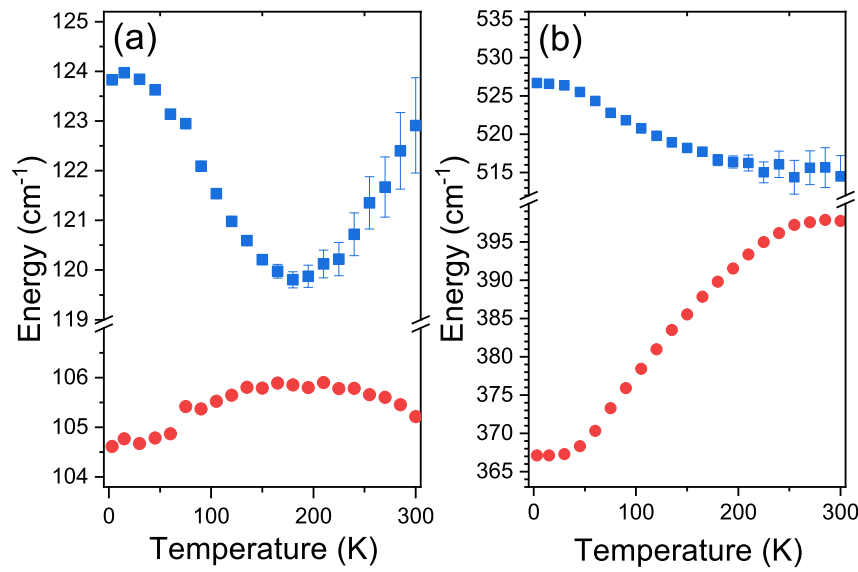

FIG. 6. Summary plots of the temperature dependence of the (a) $E_{g}$ bending phonon (red circles) and the lower energy CEF excitation (blue squares) and the (b) $A_{1 g}$ stretching phonon (red circles) and the higher energy CEF excitation (blue squares). Error bars for the fits of the spectra are included, though for most points on the summary plots they are smaller than the symbols.

nonmonotonic temperature dependence, softening in energy between 300 and $180 \mathrm{~K}$ from $\omega \approx 124$ to $120 \mathrm{~cm}^{-1}$, but hardening to $124 \mathrm{~cm}^{-1}$ as the temperature is decreased further. The higher energy CEF excitation hardens from $\omega \approx 515$ to $527 \mathrm{~cm}^{-1}$ as the temperature is reduced from 300 to $3 \mathrm{~K}$. Significantly, Fig. 6 shows that the temperature dependencies of the $124 \mathrm{~cm}^{-1} \mathrm{CEF}$ and $515 \mathrm{~cm}^{-1}$ CEF excitations track and are opposite those of the $105 \mathrm{~cm}^{-1} E_{g}$ and $396 \mathrm{~cm}^{-1} A_{1 g}$ phonon modes, respectively, suggesting possible strong coupling between these pairs of excitations in $\mathrm{Pr}_{2} \mathrm{O}_{3}$.

The strong coupling between CEF excitations and phonon excitations in rare-earth materials such as $\operatorname{Pr}_{2} \mathrm{O}_{3}$ are well known [1-3,5-8], and result from the energetic proximity of $\mathrm{CEF}$ and phonon excitations. Under these conditions one must consider the electronic CEF energy levels as being dynamically modulated by energetically proximate phonons. This situation has been described theoretically by Yamada and others using a coupled pseudospin-phonon model, in which the electronic state of the rare earth ions are represented as a stochastic "pseudospin" variable coupled to a phononic degree of freedom [20-22], with an interaction $V(k)$ given by

$$
V(k)=\frac{1}{\sqrt{N}} \sum_{j} \omega_{k} g_{k} Q_{k} \sigma_{j} e^{i k \cdot r_{j}},
$$

where $\omega_{k}$ is the bare frequency of the phonon mode with normal coordinate $Q_{k}, \sigma_{j}$ is the pseudospin operator associated with the CEF excitation at ionic site $j$, and $g_{k}$ is the pseduospin-phonon coupling coefficient.

The above pseudospin-phonon coupling model predicts a renormalization of the phonon energies by the CEF excitations, and a consequent temperature-dependent softening of the coupled phonon modes for CEF energies greater than the phonon energies [20-22], consistent with our observations for $\mathrm{Pr}_{2} \mathrm{O}_{3}$ (see Fig. 6). Interestingly, the pseudospin-phonon model also predicts that the CEF-phonon coupling should result in a structural distortion below a critical temperature de- 


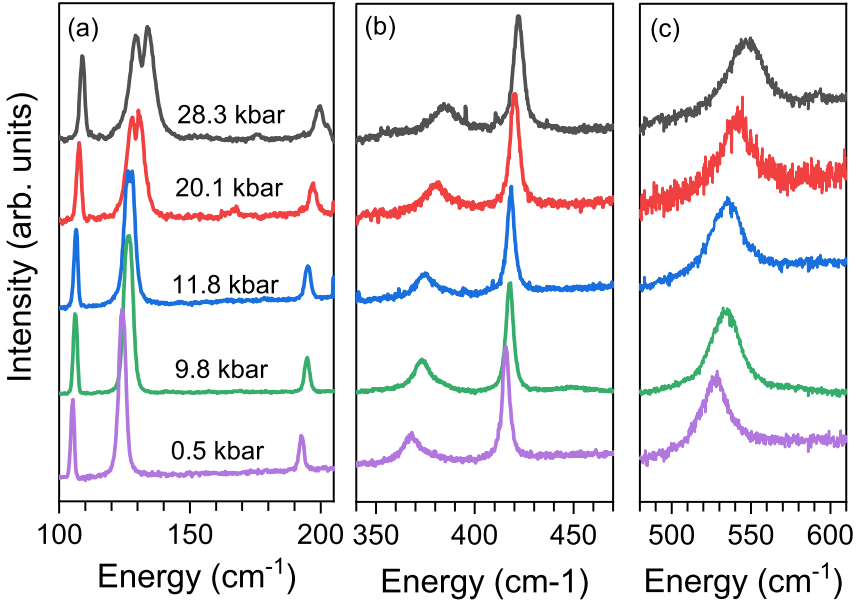

FIG. 7. Plots of the pressure dependence of Raman intensities of the zone-center phonons and CEF excitations in $\mathrm{Pr}_{2} \mathrm{O}_{3}$ showing the clear pressure-induced splitting of the lower energy CEF excitation and the significant pressure-driven hardening of the $A_{1 g}$ stretching phonon at $T=3 \mathrm{~K}$. Individual spectra are offset along the vertical axis for clarity and rescaled to maintain a consistent intensities between spectra for the most intense phonon in (a) and (b) and the higher energy CEF excitation in (c).

pendent on the coupling strength. In the coupled pseudospinphonon description, this structural distortion is concomitant with an ordering of the pseudospin variable [20-22], which could present as magnetic ordering in $\mathrm{Pr}_{2} \mathrm{O}_{3}$. We observe no Raman scattering evidence for a temperature-dependent structural transition-such as the splitting of degenerate phonon modes or the appearance of new modes-down to $3 \mathrm{~K}$ under ambient pressure conditions, although we cannot exclude the possibility of a structural transition at temperatures lower than $3 \mathrm{~K}$. Nevertheless, the soft mode behavior we observe in $\mathrm{Pr}_{2} \mathrm{O}_{3}$ suggests that $\mathrm{Pr}_{2} \mathrm{O}_{3}$ is near an incipient structural transition caused by strong CEF-phonon coupling.

\section{Pressure- and magnetic field-dependent excitation spectra of $\mathrm{Pr}_{2} \mathrm{O}_{3}$}

To further explore the importance of coupling between phonons and CEF excitations in $\mathrm{Pr}_{2} \mathrm{O}_{3}$, pressure dependent measurements were performed. The pressure-dependence of the low energy excitation spectrum of $\mathrm{Pr}_{2} \mathrm{O}_{3}$ is shown in Fig. 7. All of the phonons and CEF excitations in $\operatorname{Pr}_{2} \mathrm{O}_{3}$ increase in energy with increasing pressure, as summarized in Fig. 8. Notably, Figs. 7 and 8 show that the $124-\mathrm{cm}^{-1}$ CEF excitation splits into two distinct peaks above $10 \mathrm{kbar}$. The pressure-dependent splitting of the $124-\mathrm{cm}^{-1} \mathrm{CEF}$ excitation can be extrapolated to approximately zero at ambient pressures, implying that the splitting grows continuously with pressure. There are no observed pressure-induced changes in the phonon spectrum - such as additional phonons or a splitting of the observed Raman-active phonon modes-that indicate a substantial structural phase change.

The temperature dependence of the low energy excitation spectrum of $\mathrm{Pr}_{2} \mathrm{O}_{3}$ at high pressures is shown in Fig. 9(a), and the pressure dependence of the $124 \mathrm{~cm}^{-1}$ CEF excitation energy is summarized at various temperatures in Fig. 9(b).
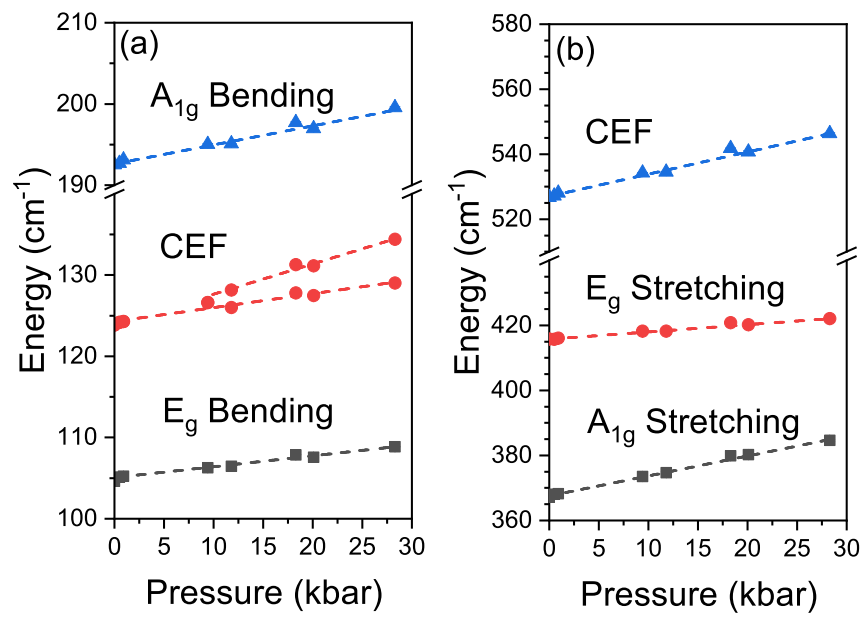

FIG. 8. Summary plots of the pressure dependencies of the energies of the Raman-active features in $\mathrm{Pr}_{2} \mathrm{O}_{3}$ based on fittings of the spectra measured at $T=3 \mathrm{~K}$. All of the Raman-active features we observe display linear pressure dependencies to their energies, and as such, linear fits of the energies of each feature are included as guides to the eye. Error bars have been omitted as they are smaller than the symbols.

Figure 9(a) shows that the pressure-induced splitting of the 124- $\mathrm{cm}^{-1} \mathrm{CEF}$ excitation becomes less clear as the temperature is increased. Note that for temperatures above $120 \mathrm{~K}$, it is ambiguous as to whether or not the splitting has disappeared completely_or is simply unresolvable_-in the high pressure spectra at these elevated temperatures; however, as discussed in the experimental section of this paper, when comparing the fits provided by one or two Lorentzian peaks, the best fits of the $120-\mathrm{K}$ and $150-\mathrm{K}$ data involve a single Lorentzian peak for the CEF excitation, suggesting that the CEF splitting disappears for $T \geqslant 120 \mathrm{~K}$. Figure 10 summarizes the temperature and pressure regions in which the pressure-induced splitting of the CEF excitation is clearly observed (red squares in Fig. 10) and not observed (blue circles in Fig. 10).

When considering the possible origins of the pressureinduced $\mathrm{CEF}$ splitting observed in the shaded region of the $\mathrm{Pr}_{2} \mathrm{O}_{3}$ phase diagram, we note that the absence of new phonons or a splitting of degenerate phonon modes in Figs. 7, 8, and 9(a) suggest that the modest pressures we have applied do not cause a significant change to the $A$-type structure of $\operatorname{Pr}_{2} \mathrm{O}_{3}$. This is consistent with previous highpressure studies of the RE sesquioxides showing that the $A$-type structure is the most stable structure [23-27], with the exception of a structural distortion observed clearly in $\mathrm{La}_{2} \mathrm{O}_{3}$ above 80 kbar [28], a significantly higher pressure than that applied in our study. In particular, high pressure x-ray diffraction and Raman scattering studies of the pressure-induced $A$-type structures of $\mathrm{Tb}_{2} \mathrm{O}_{3}$ [27] and $\mathrm{Y}_{2} \mathrm{O}_{3}$ [24,25] show that the $A$-type structure is the highest pressure structural phase observed, persisting over a wide pressure range-120-250 kbar in $\mathrm{Tb}_{2} \mathrm{O}_{3}$ [27] and 240-320 kbar in $\mathrm{Y}_{2} \mathrm{O}_{3}$ [24,25]-to the highest pressures measured.

Instead, we propose that the pressure-induced splitting of the $124 \mathrm{~cm}^{-1}$ CEF excitation is indicative of a perturbative magnetoelastic modification to the CEF Hamiltonian in 

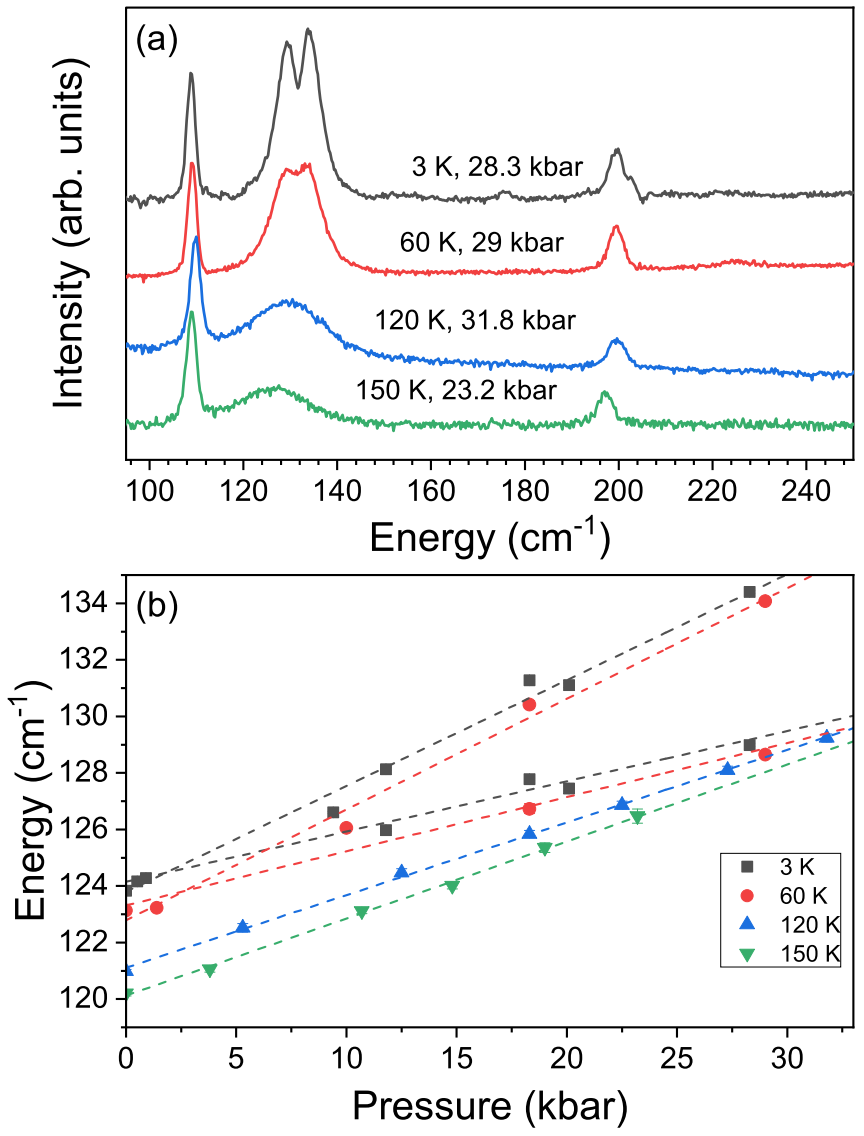

FIG. 9. (a) Low energy Raman spectra of $\operatorname{Pr}_{2} \mathrm{O}_{3}$ at the maximum pressure measured at each of the temperatures measured in the combined pressure-temperature experiments. Individual spectra have been rescaled vertically to maintain a consistent intensity for the $E_{g}$ bending phonon mode between spectra, and have been vertically offset for clarity. (b) Summary plot of the lower energy CEF excitation energies, showing the splitting of the level for $T \leqslant 60 \mathrm{~K}$ and $P>10 \mathrm{kbar}$; any possible splitting at lower pressures is not resolvable. Dashed lines are linear fittings of the energies and have been included as guides to the eye.

Eq. (1) that lifts the degeneracies of the $\Gamma_{3}$ CEF levels in $\mathrm{Pr}_{2} \mathrm{O}_{3}$ by a small uniaxial distortion [29]. We note in this regard that the pressure applied to the sample by the MCDAC is quasihydrostatic, however we expect small anisotropic pressure variations within the MCDAC. A similar pressure induced splitting of the $\left|J_{z}= \pm 1\right\rangle$ CEF levels of the hexagonal sites in metallic Pr has been observed for very modest pressures $(P<1$ kbar $)[10]$.

Measurements of $\mathrm{Pr}_{2} \mathrm{O}_{3}$ with simultaneous application of pressure and magnetic field, shown in Figs. 11(a) and 11(b), further illustrate the similar effects of magnetic field and pressure on the $\Gamma_{3}$ CEF excitation in $\mathrm{Pr}_{2} \mathrm{O}_{3}$. As summarized in Fig. 11(c), the pressure-induced splitting of the $124-\mathrm{cm}^{-1}$ CEF excitation at zero magnetic field increases roughly symmetrically with increasing field, providing further evidence that pressure splits the $\Gamma_{3}$ excited level into singlet levels having opposite angular momenta.

Lastly, if the pressure-induced splitting we observe is correlated with increased exchange coupling, the correspondence

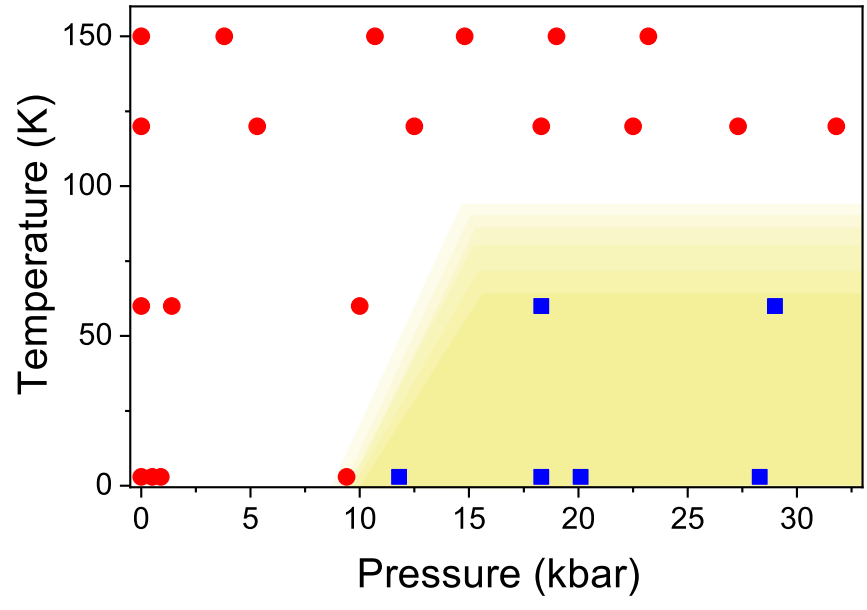

FIG. 10. Plot of the measured pressure and temperature data points. Blue squares indicate measurements which show a clear splitting of the $\Gamma_{3}$ lowest energy CEF level, while red circles indicate measurements which lack a clear splitting of the $\Gamma_{3}$ lowest energy CEF level. While pressure is expected to split the $\Gamma_{3}$ levels continuously from $P=0 \mathrm{kbar}$, the width of the peak associated with the lowest energy CEF level in our Raman spectra prevents the observation of such a splitting until it becomes sufficiently large.

between the pressure- and field-induced splittings we observe in $\operatorname{Pr}_{2} \mathrm{O}_{3}$, shown in Fig. 12, suggests that a rather modest pressure of $28 \mathrm{kbar}$ is associated with a splitting equivalent to that induced by an approximately 11-T applied magnetic field. Notably, the pressure-induced splitting of the $\left|J_{z}= \pm 1\right\rangle$ CEF levels in metallic Pr has been predicted [10], and observed [30] to be a precursor to the development of magnetic order in the material. Unfortunately, while our results provide evidence for pressure-induced magnetostructural effects in $\mathrm{Pr}_{2} \mathrm{O}_{3}$, we cannot spectroscopically confirm the onset of magnetic order in the high-pressure regime of $\operatorname{Pr}_{2} \mathrm{O}_{3}$ (shaded

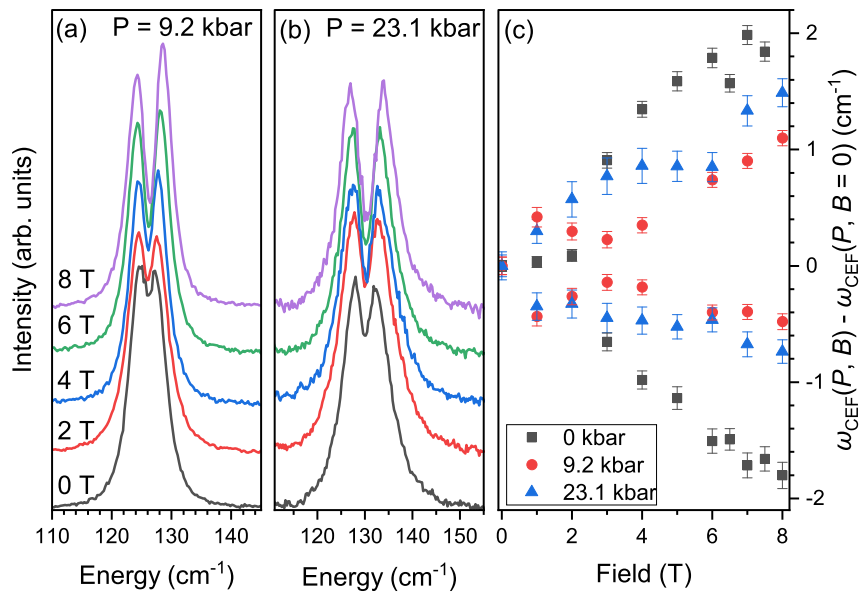

FIG. 11. Plot of the field dependent intensities of the low energy CEF excitations at (a) $P=9.2$ and (b) $23.1 \mathrm{kbar}$ at $T=3 \mathrm{~K}$. As the applied magnetic field strength is increased, we observe that the CEF excitations become more sharply resolved and the splitting between them increases. (c) Summary plot of the differences between the energies of the CEF excitations at zero field and elevated fields at the three measured pressures. 


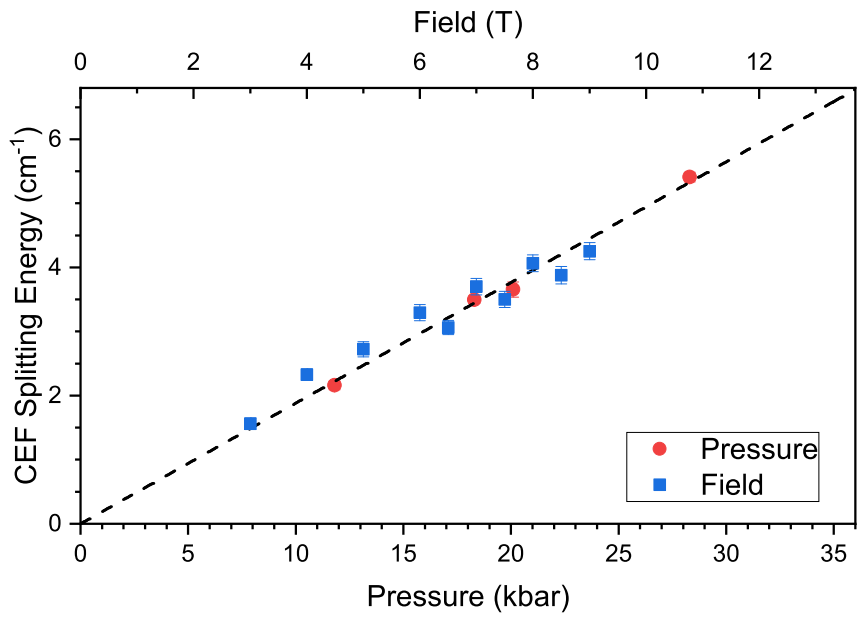

FIG. 12. Summary plot of the magnetic field and pressure dependencies of the lower energy CEF splitting at $T=3 \mathrm{~K}$. The pressure-induced splitting can be overlaid with the magnetic fieldinduced splitting, allowing for an estimation of the internal magnetic field. A fit line through the origin is provided as a guide to visually assist in correlating magnetic field strength with applied pressure.

region in Fig. 10); however, pressure-dependent magnetic susceptibility measurements would be useful for confirming the possibility of pressure-induced magnetism in $\operatorname{Pr}_{2} \mathrm{O}_{3}$.

\section{CONCLUSION}

In this study, temperature-dependent Raman scattering measurements of $\mathrm{Pr}_{2} \mathrm{O}_{3}$ reveal phonon mode softening and concomitant anomalous temperature dependencies associated with the low energy CEF excitations. We propose that the correlated and anomalous temperature dependencies of the phonons and $\mathrm{CEF}$ excitation in $\mathrm{Pr}_{2} \mathrm{O}_{3}$ result from the dynamic modulation of the CEF levels by the energetically proximate phonons. This CEF-phonon coupling and phonon mode softening is shown to be qualitatively described using the coupled pseudospin-phonon model [20-22] in which the soft phonons are strongly coupled to pseudospins representing the occupation of the low energy CEF level.

Additional evidence for strong coupling of the lattice and electronic degrees of freedom in $\mathrm{Pr}_{2} \mathrm{O}_{3}$ were observed in high pressure measurements. At high pressures the degeneracy of the $\omega=124 \mathrm{~cm}^{-1}$ CEF excitation doublet is lifted in a manner similar to the splitting induced by a magnetic field, suggesting that pressure-induced magnetostructural changes lift the degeneracy of the first excited $\Gamma_{3} \mathrm{CEF}$ level in $\mathrm{Pr}_{2} \mathrm{O}_{3}$. Interestingly, a similar pressure-induced splitting of the $\left|J_{z}= \pm 1\right\rangle$ levels of the hexagonal sites of $\mathrm{Pr}$ metal has been previously identified as a precursor to pressureinduced magnetic order [10,30]. We suggest that $\operatorname{Pr}_{2} \mathrm{O}_{3}$ shows promise for observing induced magnetic order via pressure; pressure-dependent magnetic susceptibility or inelastic neutron scattering measurements would be useful for exploring possible pressure-induced magnetism in $\operatorname{Pr}_{2} \mathrm{O}_{3}$.

\section{ACKNOWLEDGMENTS}

Research was supported by the National Science Foundation under Grant No. NSF DMR 1800982. T.K. was supported by NIMS internal projects Grants No. PA5160 and No. PA4020.
[1] M. Dahl and G. Schaack, Phys. Rev. Lett. 56, 232 (1986).

[2] R. J. Elliott, R. T. Harley, W. Hayes, and S. R. P. Smith, Proc. R. Soc. London, Ser. A 328, 217 (1972).

[3] K. Kishimoto, T. Ishikura, H. Nakamura, Y. Wakabayashi, and T. Kimura, Phys. Rev. B 82, 012103 (2010).

[4] K. Kirschbaum, A. Martin, D. A. Parrish, and A. A. Pinkerton, J. Phys.: Condens. Matter 11, 4483 (1999).

[5] H. Gerlinger and G. Schaack, Phys. Rev. B 33, 7438 (1986).

[6] K. Ahrens and G. Schaack, Phys. Rev. Lett. 42, 1488 (1979).

[7] P. Thalmeier and P. Fulde, Z. Phys. B 26, 323 (1977).

[8] A. Sethi, J. E. Slimak, T. Kolodiazhnyi, and S. L. Cooper, Phys. Rev. Lett. 122, 177601 (2019).

[9] K. N. Boldyrev, T. N. Stanislavchuk, A. A. Sirenko, L. N. Bezmaternykh, and M. N. Popova, Phys. Rev. B 90, 121101(R) (2014).

[10] K. McEwen, W. Stirling, and C. Vettier, Physica B+C 120, 152 (1983).

[11] O. Sovers, J. Lumin. 17, 93 (1978).

[12] A. Dewaele, M. Torrent, P. Loubeyre, and M. Mezouar, Phys. Rev. B 78, 104102 (2008).

[13] J. B. Gruber, B. H. Justice, J. Edgar F. Westrum, and B. Zandi, J. Chem. Thermodyn. 34, 457 (2002).
[14] O. Moune, M. Faucher, C. Jayasankar, and A. Lejus, J. Lumin. 85, 59 (1999).

[15] A. Lejus, J. Bernier, and R. Collongues, J. Solid State Chem. 16, 349 (1976).

[16] S. Kern, J. Chem. Phys. 40, 208 (1964).

[17] J. Gouteron, D. Michel, A. Lejus, and J. Zarembowitch, J. Solid State Chem. 38, 288 (1981).

[18] J. Zarembowitch, J. Gouteron, and A. M. Lejus, Physica Status Solidi (b) 94, 249 (1979).

[19] D. Mihailovic, K. F. McCarty, and D. S. Ginley, Phys. Rev. B 47, 8910 (1993).

[20] Y. Yamada, Ferroelectrics 16, 49 (1977).

[21] Y. Yamada, M. Mori, and Y. Noda, J. Phys. Soc. Jpn. 32, 1565 (1972).

[22] Y. Yamada, H. Takatera, and D. L. Huber, J. Phys. Soc. Jpn. 36, 641 (1974).

[23] H. R. Hoekstra, Inorg. Chem. 5, 754 (1966).

[24] E. Husson, C. Proust, P. Gillet, and J. Itie, Mater. Res. Bull. 34, 2085 (1999).

[25] I. Halevy, R. Carmon, M. Winterrose, O. Yeheskel, E. Tiferet, and S. Ghose, J. Phys.: Conf. Ser. 215, 012003 (2010).

[26] D. Richard, L. A. Errico, and M. Rentería, J. Alloys Compd. 664, 580 (2016). 
[27] J. Ibáñez, J. Ángel Sans, V. Cuenca-Gotor, R. Oliva, Ó. Gomis, P. Rodríguez-Hernández, A. Muñoz, U. Rodríguez-Mendoza, M. Velázquez, P. Veber, C. Popescu, and F. J. Manjón, Inorganic Chemistry 59, 9648 (2020).
[28] J. P. McClure, Ph.D. thesis, University of Nevada, Las Vegas, 2009.

[29] J. Jensen and A. R. Mackintosh, Rare Earth Magnetism (Clarendon Press Oxford, 1991).

[30] J. Jensen, J. Phys. C 9, 111 (1976). 